\title{
A SERVOANALYTIC STUDY OF CONSENSUAL PUPIL REFLEX TO LIGHT ${ }^{1}$
}

\author{
LAWRENCE STARK AND PHILIP M. SHERMAN \\ Section of Neurology and Department of Electrical Engineering, \\ Yale University, New Haven, Connecticut
}

(Received for publication July 9, 1956)

\section{INTRODUCTION}

MANy BIOLOGICAL PROCESSES, especially those involving the functioning of the central nervous system, behave as self-regulatory devices or servomechanisms. The pupil reflex to light is an example of such a process. This paper approaches the problem of a quantitative study by applying servoanalytic concepts and techniques $(3,9)$. A servomechanism is an automatic regulatory device actuated by the difference or "error" between a desired or reference input and the actual value of output. A controlled quantity is maintained dependent upon reference input despite disturbances within and external to the system. A "loop" is formed as the output quantity is fed back to the input. In Fig. 1 a block diagram of a simple servosystem resembling the pupil reflex is shown. The amount of light flux falling on the retina is the controlled quantity $\left(L_{C}\right)$. A reference light flux quantity $\left(L_{R E F}\right)$ exists

A SIMPLE SERVO SYSTEM

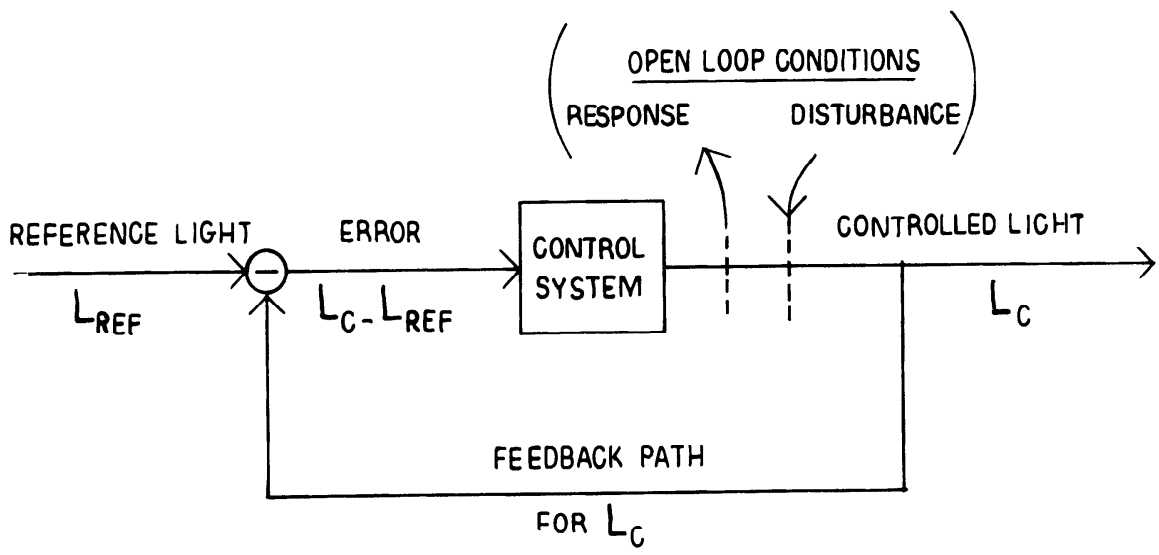

Fig. 1. A simple servosystem. This shows forward and feedback paths in the servoloop and different components therein. Symbols are explained in diagram and text. Dashed lines indicate where loop might be opened. A disturbance could be introduced and response around the loop measured.

1 This research was supported by the Neurological Research Fund of the Section of Neurology, Yale University School of Medicine. 
which is compared with actual light $\left(L_{C}\right)$ falling on the retina. Any difference or error $\left(L_{C}-L_{R E F}\right)$ which occurs is measured, and it is this quantity which actuates the control system, or pupil neuromuscular apparatus. The control system then operates, varying pupil size and so changing controlled light $\left(L_{C}\right)$ to reduce error.

It is of interest to note the historical development of these concepts in biology. The operating characteristics of the "milieu interieur" of Claude Bernard and of "homeostasis" as proposed by Cannon can be restated in such a way as to make the relationship between this development and servotheory clear (2). A homeostatic mechanism is one which senses the difference between desired and actual states and then puts into force a series of processes which in turn produces opposing effects to minimize error. A more subtle conceptual comparison is made when one transforms the reflex arc into the servoloop by adding a continuous sensory input and thus "feeds back" information concerning final state or "closes the loop" (16). The title of Charles Bell's 1826 paper, "On the nervous circle which connects the voluntary muscles with the brain," illuminates the point (1).

More recently, "nervous circles" have been further discussed $(6,7,10,15)$ and several interesting experiments have been performed. In one, Merton applied a quantitative explanation in terms of servotheory to the silentperiod phenomenon of the myotatic reflex $(11,12)$. In another, Pringle and Wilson determined the transfer function for the response of a sense organ to a harmonic stimulus (14). These and other experiments $(4,5,8,20,21)$ suggest the application of servoanalytic concepts to the study of the nervous system.

\section{APPROACH}

Experimental techniques with which a servoengineer analyzes an automatic control device are somewhat similar to methods used by the physiologist. First, the engineer draws a block diagram or an anatomical sketch of the system and its functional components, as in Figs. 1 and 4. Then he disturbs the system and traces response through the loop. This procedure requires continuous measurement of responses, as well as quantitative control over inputs. A further, more sophisticated, technique involves "opening the servoloop." We shall now discuss how these methods have been adapted to a study of the nervous system.

The pupil reflex to light has the significant advantage of being accessible in normal organisms. No dissection or surgical manipulation of the intact animal is required in order to visualize its response. We are then not forced to make the assumption that an isolated system behaves as it would in the normal state. The pupil response to light has been the subject of much study on the part of early physiologists and neurologists including Robert Whytt, Magendie, Claude Bernard, Arygll Robertson, and Horner. Recent studies have applied electrophysiological techniques and stereotaxic instruments to define more precisely pathways of the reflex. The pupil servoloop includes 
such structures as the retina, both branches of the autonomic nervous system, and the mid-brain, thus suggesting a considerable degree of complexity.

The challenge of pupil accessibility in normal organisms and its seeming ease of quantification has been the source of much activity in recent years. The most helpful of these studies to our work has been infra-red motion picture photography of the pupil as described by Lowenstein (8) and Talbot (19) and clinical researches carried out by the former author. Because of the large amount of quantitative data necessary for our proposed approach, we felt it impossible to use these photographic techniques and developed a simpler procedure. In Fig. 2, essential portions of the experimental arrangement are shown. The technical details of electrical and optical equipment

\section{EXPERIMENTAL ARRANGEMENT}

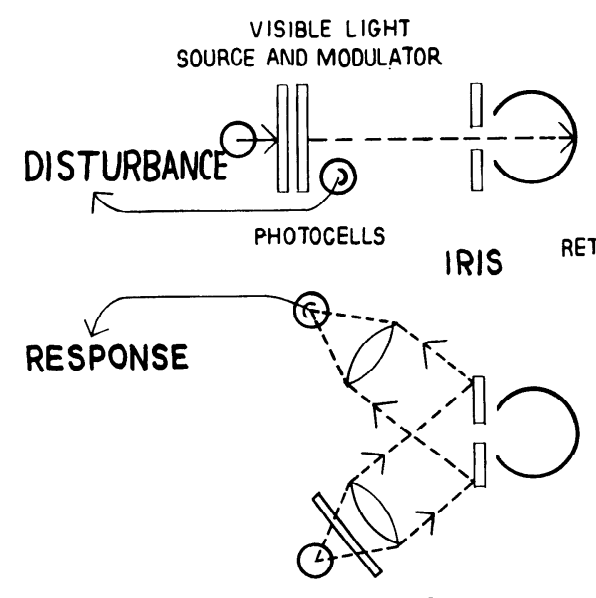

INFRARED SOURCE AND FILTER

Fig. 2. Experimental arrangement. Optical portions of visible light stimulus path are not shown. Modulator consisted of polaroid filters in rotary oscillation with respect to each other. A fixation point was provided, as well as a biteboard.

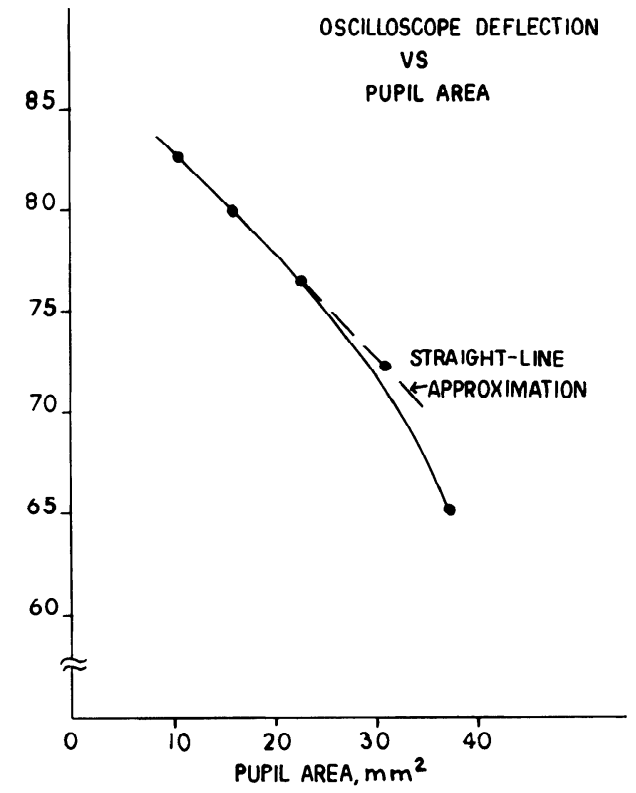

FIg. 3. Response calibration. Response photocell current is shown in arbitrary units. Pupil area was determined from diameter measurements, from enlarged photographs. Each point is average of two or three flash photographs taken at same light intensity.

will be described fully elsewhere (17). Pupil area was measured continuously by reflecting an infra-red light from the iris to a photocell. When the pupil was large and the iris small, less infra-red was reflected onto the photocell. Conversely, when the pupil was small and the iris large, more infra-red was reflected onto the photocell. Thus we had a convenient and continuous measurement of system response. Figure 3 shows a calibration of the reflection method for measuring pupil area. Divergence from linearity was small 
over the experimental range and it was not necessary to correct for this.

Figure 2 shows how the stimulus was quantitatively manipulated in a specific manner. A visible light source was directed onto the retina of a human subject, who rested on a biteboard and looked at a dim, distant, fixation point. It was not found mechanically feasible to arrange all the apparatus in front of one eye, and therefore the consensual reflex was studied. In experiments described below, intensity of light source was sinusoidally modulated by means of two polaroid filters in reciprocal oscillation with respect to each other. A portion of the stimulus light beam was continuously sampled by a photocell, calibrated in terms of actual light intensity hitting the cornea. There were three reasons for our use of sinusoidal stimuli: (i) experimental techniques for obtaining a given level of accuracy are simple, (ii) the mathematical analysis is well understood and relatively easy to manipulate, and (iii) system design and performance are evaluated readily. As an example of the first point, once the retina had adapted to mean light intensity, we were able to vary sinusoidal modulation over the entire frequency range while the pupil system remained in a steady state.

As intensity increases, the pupil contracts; the resultant effect on light flux falling on the retina can be resolved into two factors. The first is an increase in light flux due to increase in stimulus intensity. The second is a decrease in light flux due to decrease in pupil area. System gain is defined as the ratio of the second to the first; it is dimensionless since both factors are in terms of light flux. In calculating the first factor, the change in stimulus passing through the pupil is multipled by pupil area. Since fluctuation in area is small compared to total area, we approximate by using the value of average area. Similarly, in calculating the second factor, change in pupil area is multipled by the intensity of light. Again, since fluctuations in intensity are small compared to total intensity, we approximate by using the value of average intensity. These two approximations simplify greatly the quantitative analysis and show the value of the small-signal approach. The position in the block diagram at which this linearization is effected is shown in Fig. 4.

An important "dissection" technique is widely used by the servoengineer. The system is studied under "open loop" operating conditions. This method is indicated in Figs 1 and 4 by dashed lines representing a break in the servoloop. A disturbance is injected, transmitted around the loop, and measured at the point of break. Thus system response has no influence over the disturbance, which remains entirely in the control of the experimenter. This simplifies the input-output relation, that is, the transfer function. For this reason an important aspect of the experimental approach to the pupil reflex was the development of a method to study the system in "open loop" operating condition. Provision was made for careful focusing of stimulus light so that the entire light source entered the pupil in the form of an image of a small disc whose diameter was smaller than the smallest diameter of the pupil. Changes in pupil size could then have no effect on flux hitting the 
A LINEARIZED APPROXIMATION TO THE PUPILLARY

SERVO SYSTEM

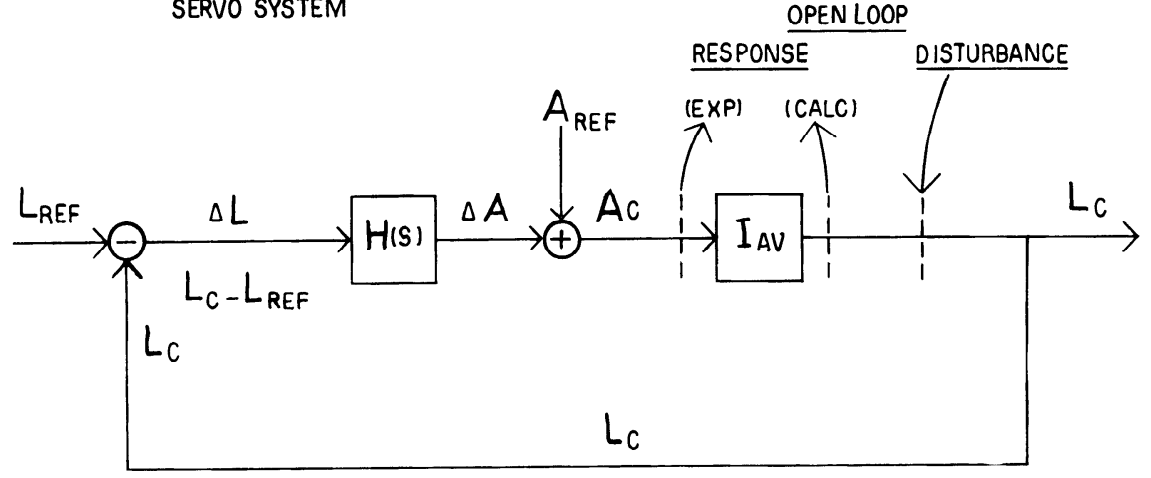

FIG. 4. Linearized approximation to pupil servosystem. Actual pupil system is more complicated than this figure indicates. However, we linearized by using small variations about a fixed operating point. Thus the necessary calculations are simplified and linear servoanalytic methods may be applied. $A_{R E F}$ is reference area, $A c$ is controlled area, $\Delta A$ is a change in area generated by control system whose transfer function is $H(s), I_{A V}$ is average intensity value used to multiply controlled area to yield controlled light flux. This approximation is explained in text.

retina. ${ }^{2}$ In calculating the first factor of system gain in "open loop" operating condition, the changing pupil area is multiplied by light intensity considered as if light flux were distributed over the entire pupil area. This supposes that the retina cannot distinguish between different light distributions at the plane of the pupil. It is not necessary to use the small-signal approximation for the first factor in this instance. In addition, when these "open loop" conditions were not required, the beam could be defocused. Then the pupil reflex could modify light flux to the retina in the usual way for "closed loop" operation.

In summary, the experimental procedure was designed to adapt servoanalytic methods to study the pupil reflex. Sinusoidal light stimuli of varying frequency were applied. The sinusoidal response was measured and its amplitude and phase relationship to the stimulus was determined. Data were obtained in both "open loop" and "closed loop" operating conditions.

\section{Experimental Results and Analysis}

A discussion of a sample experiment as shown in Fig. 5 would perhaps aid in understanding our experimental approach and the results obtained. The two oscillatory traces represent plots on a dual-beam cathode-ray oscilloscope of photocell current fluctuation as a function of time. The upper trace (a) shows stimulus intensity varying sinusoidally at a frequency of 1.3 c./sec. and upward deflection indicates decrease in light intensity. The lower trace (b) shows pupil area changing at the same frequency but approxi-

${ }^{2}$ Light scattered in the anterior chamber is still controlled by pupil area in its passage to the retina. Although this error is not significant in magnitude we hope to further evaluate it by "opening the loop" using other methods. 


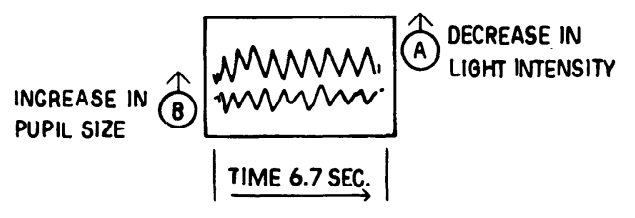

Fig. 5. Typical experimental data. A: Stimulus intensity varying sinusoidally at a frequency of $1.3 \mathrm{c}$./sec. Upward deflection indicates decrease in light intensity. B: Pupil area changing at the same frequency but approximately $180^{\circ}$ behind stimulus. Upward deflection indicates increase in pupil size. Note variability in response and harmonic distortion. Time proceeds toward right.

mately $180^{\circ}$ behind the stimulus fluctuation, i.e., upward deflection here indicates increase in pupil size. From these traces and the calibrations as explained previously, relative amplitudes of stimulus and response can be determined as well as the phase relationship between them. The phase shift is measurable in degrees and can be used directly as will be shown. Harmonic distortion contributed an error of up to 10 per cent in the phase shift measurement.

Table 1. Gain and phase data for frequency response functions

\begin{tabular}{ccc}
\hline Frequency in c./sec. & Gain (dimensionless) & Phase angle in degrees \\
\hline 0.14 & 0.16 & 60 \\
0.7 & 0.15 & 90 \\
0.9 & 0.12 & 140 \\
1.3 & 0.13 & 190 \\
1.34 & 0.11 & 190 \\
2.1 & 0.06 & 320 \\
2.3 & 0.07 & 380 \\
2.4 & 0.05 & 420 \\
2.7 & 0.05 & 470 \\
3.0 & 0.02 & 510 \\
3.4 & 0.02 & 530 \\
0.4 & - & 50 \\
1.0 & - & 180 \\
1.4 & - & 200 \\
1.6 & - & 270 \\
1.7 & - & 270 \\
\hline
\end{tabular}

Table 1 gives the results of a series of experiments run on one individual at different times under similar open-loop operating conditions. Although closed-loop frequency responses were obtained, as well as step-input responses, our analysis is based on open-loop experiments. The other results will be used to corroborate various points in the analysis (18). The data from Table 1 are plotted in Fig. 6, as the open-loop frequency response. One can readily see certain qualitative features: low gain, steep attenuation of gain at higher frequencies, and large phase shift.

It is desirable, however, to have a full but concise mathematical description of system behavior. Such a canonical expression is the "transfer function," for convenience, written as a function of the "complex frequency" operator, "s." In Fig. 4 the loop elements which form the open-loop transfer 
Fig. 6. Open loop frequency response. Amplitude is plotted on log-log scale while phase shift (lag) is on log-linear scale. Points are experimental from Table 1 and continuous lines are fitted. Dashed lines are asymptopes.

function $G(s)$ are shown. Included are $H(s)$, the transfer function relating area change output to light change input, and the linearized intensity multiplication factor $I_{A V} . G(s)$ is independent of actual break point provided only that the response has traveled completely around the loop to the point of injected disturbance.

The data displayed in Fig. 6 will now be used to derive the open-loop transfer function, $G(s)$. Low-frequency gain is 0.16 . The attenuation curve appears to have an asymptotic slope

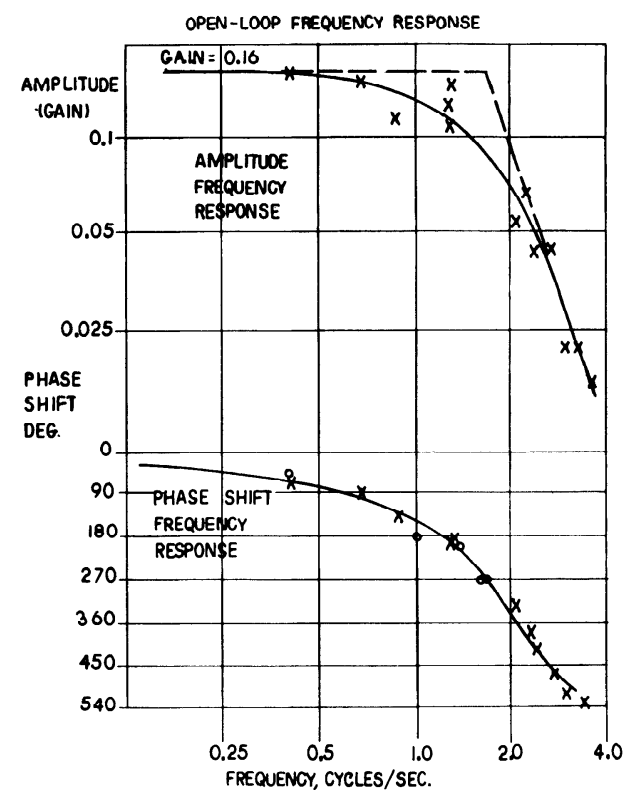
of 18 decibels per octave beyond the break frequency. This slope can be represented by three time-lag factors. The actual values of the time constants are hard to determine accurately from the present experimental data, but all apparently fall within the range of $0.05-0.2 \mathrm{sec}$. As a rough approximation we set each equal to 0.1 sec. These time lags account for $270^{\circ}$ of "minimum" phase shift at higher frequencies (for example, 4 c./sec.). By referring to the phase shift portion of Fig. 6 it can be seen that the actual phase shift is $540^{\circ}$ at this frequency. The remaining $270^{\circ}$ of "non-minimum" phase shift can be accounted for by a time delay of 0.18 sec., expressed as $e^{-018 s}$. These calculations now enable us to write the transfer function

$$
G(s)=\frac{0.16 e^{-0.18 s}}{(1+0.1 s)^{3}}
$$

Several parameters in the open-loop transfer function can be compared with data obtained from closed-loop and step-input experiments (18). The closed-loop transfer function $F(s)$ is related to $G(s)$ by the expression

$$
F(s)=\frac{G(s)}{1+G(s)} .
$$

Low-frequency closed-loop gain is calculated to be 0.14. Closed-loop gain was experimentally determined at several operating points as 0.15 . Time delay was measured in step-function experiments and found to be $0.18 \mathrm{sec}$. The agreement shown here is satisfactory, especially considering our difficulty in achieving experimentally a good small-signal approximation (e.g., 10 
per cent modulation in open-loop experiments). Furthermore, since the eye is a non-linear system, the values of constants obtained for the transfer function will depend upon operating point. Undoubtedly if other intensity levels had been used, somewhat different results would have been obtained. However, the form of the function would probably be consistent.

Another method of displaying the system behavior is the Nyquist diagram in Fig. 7. This vector plot of gain and phase angle is often used to show clearly the desired characteristics of a servomechanism--speed and accuracy of response, provided that the response is achieved in a stable manner. This means the system must not oscillate excessively as it attempts to correct for error. Other characteristics of interest are degree of stability and range of frequencies over which the system will respond.

Enclosure of the critical point at $180^{\circ}$ phase shift and unity amplitude by the curve in the Nyquist diagram indicates system instability and pre-

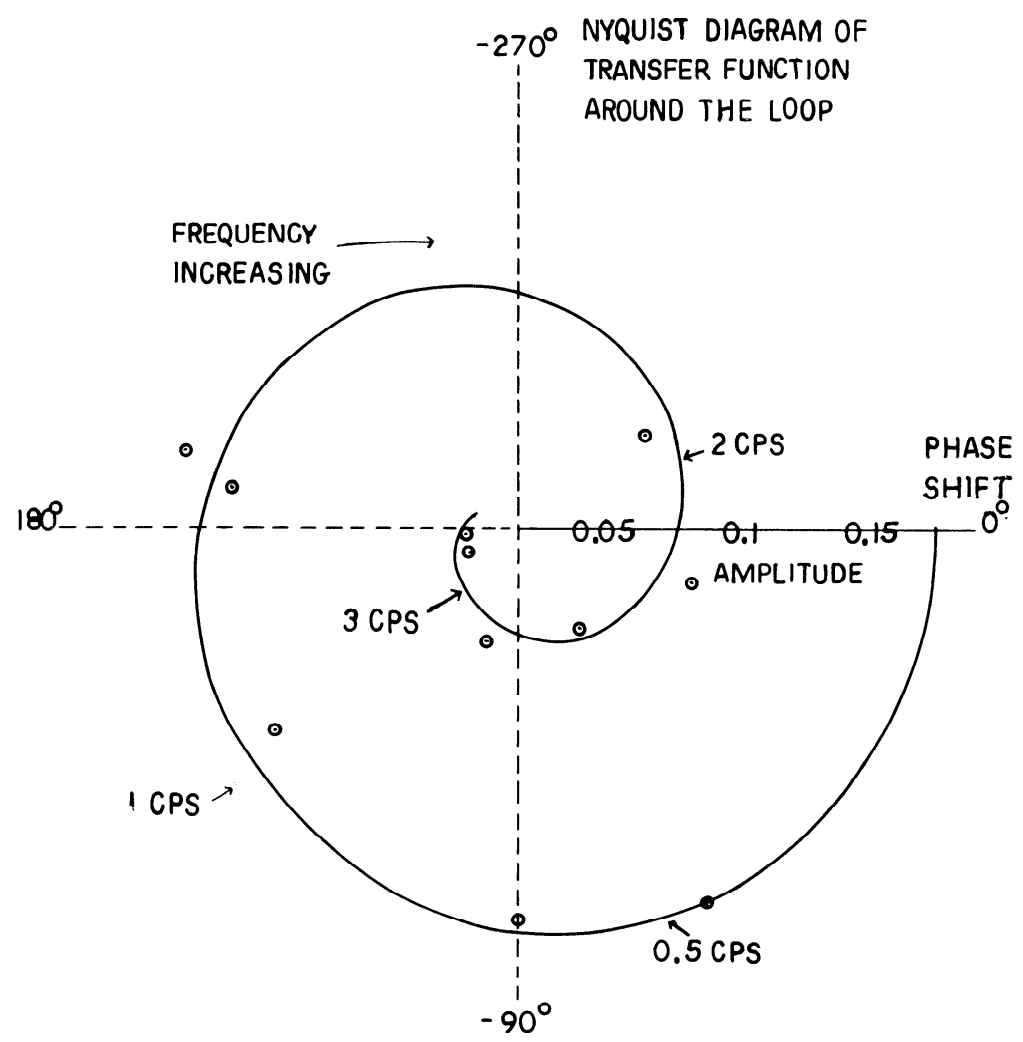

Fig. 7. Nyquist diagram of the transfer function around the loop (open). Vector plot of gain (amplitude) and phase shift. Dimensionless scale of modulus is shown. A few frequencies are indicated. Line is derived from fitted lines of Fig. 6, while points are experimental. It is to be especially noted that gain at $180^{\circ}$ phase shift is only 0.12 . This indicates that system is very stable. 
dicts sustained or divergent oscillation in response to any disturbance. The fact that the curve in Fig. 7 does not enclose the critical point indicates that the system is stable. Furthermore, the degree of stability of the system can be determined by the distance of the curve from the critical point. Since the gain is 0.12 at $180^{\circ}$ phase shift, the pupil system is clearly very stable.

\section{Discussion}

It is not surprising that the data presented here fall into the pattern of servoanalysis, since the pupil reflex is a servomechanism by definition, i.e., it is an error-actuated loop. The accessibility of the pupil to quantification is almost unique. Despite this, we have made only a crude beginning in applying the methods of servoanalysis in neurology. However, there were no comparable studies of biological control devices which could have offered guidance in this work. The pupil system certainly warrants further investigation to clarify various aspects that we have found difficulty in treating. The system also serves as a model of other servoloops in neurophysiology.

Oscillation is a common phenomenon that can be interpreted in the same terms for both engineering and biological self-regulatory devices. It indicates malfunctioning of a servomechanism due to excessive gain and phase lag. It is also a frequent pathological abnormality in neurological diseasesfor example, tremor, clonus, ataxia, nystagmus and hippus. Further study in this direction would be rewarding.

Various factors in the transfer function, $G(s)$, can be assigned to specific elements comprising the pupil loop. A correlation of this type could be used to define the mechanism of each individual element. This definition could be used as a guide in studying the particular activities of an element that gives it its important physiological properties. Several types of biological modifications (from physiology, pharmacology, clinical and experimental neurology) might be used to change the state of the servoloop and thus permit further analysis of the system. Conversely, quantification of these biological modifications may be obtained by studying their effects on the transfer function of the system.

\section{SUMmARY}

1. A serovanalysis of the consensual pupil reflex to light has been performed and a transfer function obtained.

2. The pupil servosystem has a very low gain and is extremely stable.

3. The quantitative experimental and analytic techniques used have been described.

4. The appropriateness of this investigative method for studying the nervous system has been explained.

\section{ACKNOWLEDGEMENTS}

The authors wish to thank Professors Tom N. Cornsweet, Gilbert H. Glaser and Peter M. Schultheiss for advice, encouragement and support. 


\section{REFERENCES}

1. BeLL, C. On the nervous circle which connects the voluntary muscles with the brain. Proc. Roy. Soc., 1826, 2: 266-267.

2. Cannon, W. B. The wisdom of the body. New York, W. W. Norton, 1932, 312 pp.

3. Chestnut, H. ANd Mayer, R. W. Servomechanisms and regulating system design. New York, John Wiley and Sons, 1951, vol. 1.

4. Cornsweet, T. N. Determination of the stimuli for involuntary drifts and saccadic eye movements. J. opt. Soc. Amer., 1956, 46: 987-994.

5. Fitss, P. M. Engineering psychology and equipment design. Chap. 35 in: Stevens, S. S., ed. Handbook of experimental psychology. New York, Wiley, 1951.

6. Kubik, L. A theoretical application to some neurological problems of the properties of excitation waves which move in closed circuits. Brain, 1930, 52: 166-185.

7. LoREnte de Nó, R. Analysis of the activity of chains of internuncial neurons. J. Neurophysiol., 1938, 1: 207-231.

8. Townstern, O. Pupillary reflex shapes and topical clinical diagnosis. Neurology, 1955, 5: 631-644.

9. MacMillan, R. H. Theory of control in mechanical engineering. Cambridge, Cambridge Univ. Press, 1951. 195 pp.

10. McCulloch, W. S. Why the mind is in the head. Pp. 42-57 in: Cerebral mechanisms in behavior, 'The Hixon Symposium. New York, Wiley, 1951.

11. Merton, P. A. The silent period of a muscle in the human hand. J. Physiol., 1951, 114: $183-198$.

12. Merton, P. A. Speculations on the servocontrol of movement. Pp. 247-260 in: The spinal cord, a Ciba Foundation symposium. London, Churchill, 1953.

13. Nyquist, H. Regeneration theory. Bell System tech. J., 1932, 11: 126-147.

14. Pringle, J. W. S. and Wilson, V. J. The response of a sense organ to a harmonic stimulus. J. exp. Biol., 1952, 29: 220-234.

15. Ranson, S. W. And Hinsey, J. C. Reflexes in the hind limbs of cats after transection of the spinal cord at various levels. Amer. J. Physiol., 1930, 94: 471-492.

16. Sherrington, C. S. The integrative action of the nervous system. London, Constable, 1909, $393 \mathrm{pp}$.

17. Stark, L., Cornsweet, T. N., Sherman, P. M. and Schultheiss, P. M. A new stimulating and recording pupillometer. 1956 (In preparation).

18. Stark, L. ANd Sherman, P. M. 1956 (Unpublished experiments).

19. Talbot, S. A. Pupillography and pupillary transient. Ph.D. Thesis, Dept. Physics, Harvard University, January 1938.

20. VAn Der Tweel, L. Reaction of the pupil. Ph.D. Thesis, Dept. Ophthalmology, University of Amsterdam, 1956.

21. Westheimer, G. The mechanism of saccadic eye movements. Arch. Ophthal., 52: 1954. $710-724$. 